\title{
Control of hybrid AC/DC microgrid under islanding operational conditions
}

\author{
Guangqian DING, Feng GAO (ه), Song ZHANG, \\ Poh Chiang LOH, Frede BLAABJERG
}

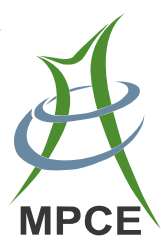

\begin{abstract}
This paper presents control methods for hybrid AC/DC microgrid under islanding operation condition. The control schemes for AC sub-microgrid and DC sub-microgrid are investigated according to the power sharing requirement and operational reliability. In addition, the key control schemes of interlinking converter with DC-link capacitor or energy storage, which will devote to the proper power sharing between AC and DC sub-microgrids to maintain $\mathrm{AC}$ and $\mathrm{DC}$ side voltage stable, is reviewed. Combining the specific control methods developed for AC and DC sub-microgrids with interlinking converter, the whole hybrid AC/DC microgrid can manage the power flow transferred between sub-microgrids for improving on the operational quality and efficiency.
\end{abstract}

Keywords Microgrid, Islanding operation, Distributed generation, Hybrid AC/DC microgrid, Inverter, Power flow

\section{Introduction}

The traditional power systems are changing globally, and a large number of distributed generation (DG) units are integrated into distribution power grid driven by the

CrossCheck date: 17 June 2014

Received: 18 June 2014 / Accepted: 23 July 2014/Published online: 19 August 2014

(C) The Author(s) 2014. This article is published with open access at Springerlink.com

G. DING, F. GAO, Key Laboratory of Power System Intelligent Dispatch and Control (Shandong University), Ministry of Education, Jinan 250061, China

$(\bowtie)$ e-mail: fgao@sdu.edu.cn

S. ZHANG, State Grid of China Technology College, Jinan,

China

P. C. LOH, F. BLAABJERG, Department of Energy

Technology, Aalborg University, Aalborg, Denmark environmental concerns and economical factors [1]. However, both utility grid and power consumers will suffer severe power quality problems as the consequence of high penetration DG units integrated without autonomous control capability. In order to overcome the inherent limitation of distributed generation concept, microgrid as a new concept has then been proposed to well manage the local DG units and loads.

Generally, the AC microgrid with high penetration of DG units, and storage devices [2-4], has more capacity and control flexibilities to be connected to the conventional AC power systems under grid-tied and islanding operation conditions. However, some new energy sources in the microgrid, such as photovoltaic (PV) panels, batteries and fuel cells are DC sources in nature, which unavoidably require inverters to be connected to grid. Meanwhile, more DC loads, e.g., LED lighting and computers etc., are rapidly growing at the end users, which need the power factor correctors (PFCs) to convert the standard AC voltage to a desired DC voltage. Intuitively, establishing a DC power supply network to connect the DC sources and DC loads directly using high efficient DC/DC converters could reduce the unnecessary power conversion circuit and simplify the control complexity. Doing so, the DC grid can demonstrate its significant characteristics with high efficiency and low power conversion cost.

The DC microgrid was proposed in [5-9] to integrate various distributed generators. In practice, several DC microgrid pilot projects, such as low voltage DC microgrids at the Italian Research Center on Energetic Systems and the Lawrence Berkeley National Laboratory, have been established and tested with $10 \%$ energy savings for their data center compared to a very efficient AC baseline case. Compared with AC microgrid, DC microgrid has other advantages of less energy conversion links, lower line losses and higher system efficiency.

In addition, the DC microgrid does not need to track the phase and frequency of $\mathrm{AC}$ voltage unlike the traditional grid- 
tied inverters, which greatly influence the controllability and the reliability of AC microgrid. Therefore, DC microgrid is more suitable for the integration of distributed renewable energy sources. However, for a comprehensive microgrid, where the various sources as complementary should be integrated to overcome the environmental influence and reduce the interruption maintenance time, a pure DC grid would be deemed inappropriate. Therefore, a hybrid AC/DC microgrid should be assumed to fully demonstrate the advantages of $\mathrm{AC}$ and DC distribution networks in view of easier renewable energy integration, higher power conversion efficiency, less energy storage capacity, and higher reliability. For example, a hybrid AC/DC microgrid project in Dongfushan Island built by State Grid Corporation of China integrated multiple distributed generation resources and energy storage and improved the power supply reliability of whole island.

In general, the advantage of hybrid AC/DC microgrid can be summarized as follows $[10,11]$ :

1) The elimination of unnecessary DC-AC or AC-DC$\mathrm{AC}$ power conversion circuits installed in the power supplies, meaning the significant reduction of power conversion losses;

2) The elimination of embedded PFCs for powering DC loads in the traditional AC grid, meaning the significant cost and loss reduction of power electronics equipment at end users;

3) The improved power quality in AC grid since the DC loads will not directly generate harmonics pollutions and the interlinking converters with full controllability can significantly enhance the power quality;

4) The improved unsymmetrical current control capability since negative and zero sequence current problems caused by the unbalanced loads in AC grid can be handled by the DC grid.

In this framework, this paper reviews the control schemes of hybrid AC/DC microgrid under islanding operational condition. This work starts with a discussion about the operational mode classification of microgrid according to the power flow in hybrid AC/DC microgrid. Based on the classification, operational features and detailed control schemes of AC sub-microgrid, DC submicrogrid and interlinking converter in hybrid AC/DC microgrid are reviewed and discussed.

\section{Classification of different operation modes in a hybrid AC/DC microgrid}

Generally, a hybrid AC/DC microgrid consists of three main parts: 1) AC sub-microgrid, 2) DC sub-microgrid and 3) power electronics interfaces between AC and DC buses.
Figure 1 shows that a general architecture of hybrid AC/DC microgrid is connected to a utility AC grid, where the DC sub-microgrid is connected to AC grid through an interlinking converter. Since the AC microgrid can be directly connected to utility grid through a simple circuit breaker, the $\mathrm{AC}$ sub-microgrid is generally dominant in the hybrid $\mathrm{AC} /$ DC microgird to provide a stable voltage. The AC power generators, such as wind turbine and small diesel generator, and the AC loads, such as AC motors and traditional lighting can connect to the AC sub-microgrid. On the other hand, DC power sources such as photovoltaic panels, fuel cells and batteries can be connected to DC sub-microgrid through simple DC/DC converters. Besides, the AC loads have variable frequency operation requirements, such as adjustable speed motors could be connected to DC sub-microgrid either. The energy storage can be installed in AC sub-microgrid or DC sub-microgrid or inserted in the interlinking converter, whose installation location should be optimized by considering load types, power flow, operational reliability, and cost. The energy storage optimization is out of the scope, which will not be discussed further.

Considering the configuration of hybrid AC/DC microgrid, three different operational modes and their power flow patterns are listed in Table 1, where Modes (a) and (b) represent the independent operation of AC and DC submicrogrids, respectively, and Mode (c) refers to the joint operation of AC and DC sub-microgrids. In specific, when AC sub-microgrid operates independently, the control targets are to properly control the AC current or AC voltage to manage the real and reactive power flow during grid-tied or islanding operational conditions. While DC sub-microgrid operates independently, the DC voltage or DC current should be carefully controlled to dispatch the power flow between DC sources and DC loads. At last, the joint operation of $\mathrm{AC}$ and $\mathrm{DC}$ sub-microgrids can properly manage the power flow between $\mathrm{AC}$ and DC networks to optimize the operational efficiency and stability. Detailed review will be presented in the following sections.

\section{Control schemes of AC sub-microgrid}

The distributed energy sources can connect to the AC submicrogrid through the interfacing converters. Therefore, the DGs can be treated as AC voltage sources or current sources operated in parallel. The load power sharing among the parallel converters under islanding operation condition has been an active research topic [12-14]. In general, to fully utilize the capacity of DGs, the output power of DGs should be proportional to their rated power. To achieve this control goal, many power sharing control schemes have been proposed.

The centralized control scheme proposed in [15] used a central controller to produce and deliver the current 


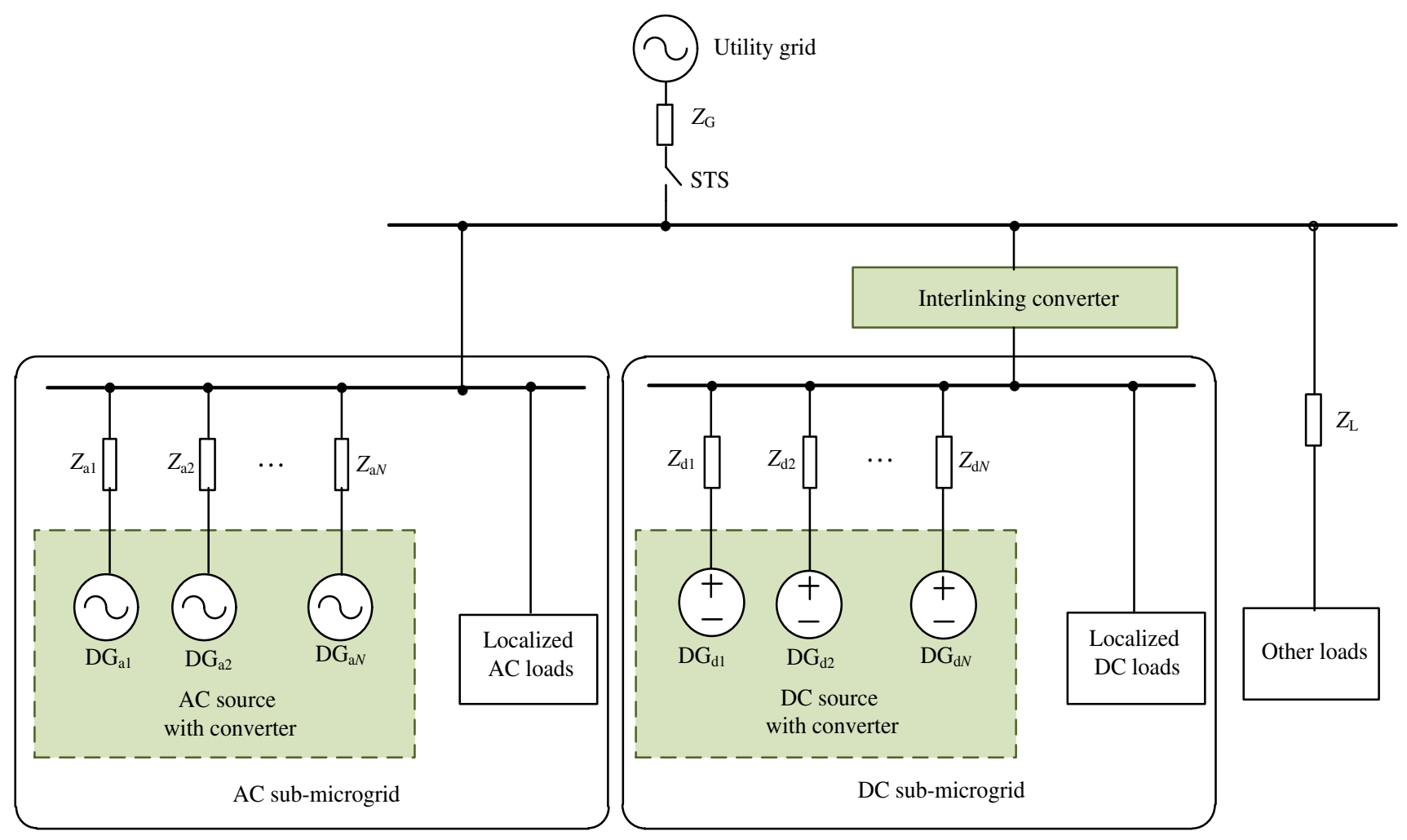

Fig. 1 A general structure of hybrid AC/DC microgrid

Table 1 Operations of hybrid AC/DC microgrid

\begin{tabular}{lllll}
\hline Operation modes & $\begin{array}{l}\text { AC sub-microgrid } \\
\text { sources }\end{array}$ & $\begin{array}{l}\text { DC sub-microgrid } \\
\text { sources }\end{array}$ & Power flow & Control system \\
\hline Mode (a) & $\sqrt{ }$ & $\times$ & $\begin{array}{c}\text { AC microgrid sources } \rightarrow \text { AC loads } \\
\text { and grid }\end{array}$ & $\begin{array}{c}\text { Control of AC voltage/AC current } \\
\text { for AC load sharing }\end{array}$ \\
Mode (b) & $\times$ & $\sqrt{\text { DC microgrid sources } \rightarrow \text { DC loads }}$ & $\begin{array}{c}\text { Control of DC voltage/DC current } \\
\text { for DC load sharing }\end{array}$ \\
Mode (c) & $\sqrt{ }$ & $\sqrt{ }$ & AC and DC microgrid sources $\rightarrow$ & $\begin{array}{c}\text { Control of AC and DC voltage } \\
\text { for AC and DC load sharing }\end{array}$ \\
\hline
\end{tabular}

reference of each DG so that all DGs can generate proper real and reactive powers simultaneously to maintain the grid stable. Master-slave control method in [16] proposed a combination of one master converter with voltage control capability and several slave converters with only current control capability like the traditional grid-tied converters. In specific, the voltage-controlled converter acts as the main converter to establish the voltage reference for other current-controlled converters, while the slave converters track the voltage reference to inject the dispatched real and reactive power. The master converter should have a relatively large capacity to fast establish the grid voltage during the grid transient interval when the current controlled converters stop injecting power to microgrid due to the loss of stable voltage. An improved method called the circular-chain-control (3C) strategy was presented in [17], where the parallel converters were cascade connection and each converter generated the current reference for its adjacent converter. Doing so the reliability of whole control system will be improved. In [18], the average current control scheme was proposed, where the current references were generated and delivered to each converter by means of communication. Although the above summarized control schemes can achieve the steady and dynamic performances of AC microgrid under islanding operational condition, the stability of the control system highly relies on the effective communications, which would reduce the operational reliability and increase the maintenance cost.

Considering the distributed source configuration in microgrid, the decentralized control method without mutual communication is more reliable for the islanding operation. 


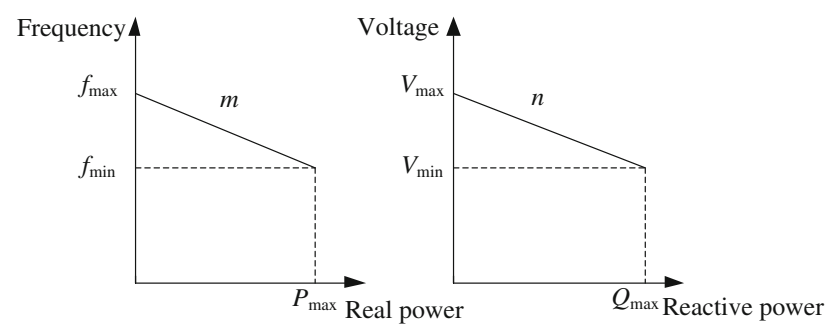

Fig. 2 Real and reactive power droop characteristics for AC submicrogrid

Therefore, the droop control method known as the independent, autonomous, and wireless control scheme is studied due to the elimination of intercommunication links among distributed converters. The main idea of droop control is to imitate the behavior of a synchronous generator, which reduces the frequency when the real power increases [19]. This control scheme can regulate the frequency and the magnitude of voltage reference in order to achieve proper power sharing. The droop control scheme can regulate the output voltage frequency and amplitude in theory as

$\left\{\begin{array}{l}f=f_{\max }-m\left(P-P_{\max }\right) \\ V=V_{\max }-n\left(Q-Q_{\max }\right)\end{array}\right.$

where $f_{\max }$ and $V_{\max }$ are the maximum DG unit output voltage frequency and amplitude without load; $m$ and $n$ refer to the coefficients of $f-P$ and $V-Q$ droop control; $P$ and $Q$ are the instantaneous real and reactive powers after low pass filter (LPF); $P_{\max }$ and $Q_{\max }$ are the maximum real and reactive powers of distributed sources, respectively; $P_{\max }$ and $Q_{\max }$ are usually set to zero when the microgid operates in islanding mode.

The droop coefficients $m$ and $n$ are determined based on the converter power rating, the maximum allowable voltage and frequency deviations, as shown in Fig. 2. The droop coefficients $m$ and $n$ are calculated as:

$\left\{\begin{array}{l}m=\frac{f_{\max }-f_{\min }}{P_{\max }} \\ n=\frac{V_{\max }-V_{\min }}{Q_{\max }}\end{array}\right.$

where $f_{\min }$ and $V_{\min }$ are the minimum allowable operating values of the voltage frequency and the amplitude, respectively.

To share the load demand in islanding microgrid according to DG ratings, the droop coefficient values shall be adjusted in inverse proportion to their rated power [20]. For the islanding microgrid with parallel inverters, the droop coefficients should follow the relationships:

$\left\{\begin{array}{l}m_{1} P_{\max 1}=m_{2} P_{\max 2}=\cdots=m_{N} P_{\max N} \\ n_{1} Q_{\max 1}=n_{2} Q_{\max 2}=\cdots=n_{N} Q_{\max N}\end{array}\right.$ where $m_{1}$ to $m_{N}$ and $n_{1}$ to $n_{N}$ are the droop coefficients of DG units 1 to $N, P_{\max 1}$ to $P_{\max N}$ and $Q_{\max 1}$ to $Q_{\max N}$ are the rated real power and reactive power of DG units 1 to $N$, respectively.

When assuming the above droop control scheme, the line impedance between distributed converter and point of common coupling (PCC) will influence the accuracy of power sharing. In [21], a virtual output impedance loop added in the droop control system was assumed to solve the problem. In principle, the added virtual impedance will force the equivalent impedance be highly inductive so that the traditional droop control theory can still work effectively. However, when directly implementing the droop control scheme in grid-tied operation condition, it will produce the frequency deviation resulting in the loss of synchronization. In [22], a hierarchical control scheme was proposed where three control levels were defined: primary control, secondary control and tertiary control. In specific, the primary control is indeed a droop control method, including an output virtual impedance loop and the secondary control allows the restoration of deviations produced by the primary control and the tertiary control manages the power flow between the microgrid and the utility grid. The hierarchical control architecture needs communication between distributed sources and central controller and it can be implemented in both grid-tied and islanding operational conditions. Because the droop control method is assumed in the bottom level controller, the controller reliability can still be guaranteed.

\section{Control schemes of DC sub-microgrid}

With the rapid increase of DC power generators and DC loads, the DC microgrid has been investigated for many years. The DC sub-microgrid can be treated as a pure DC microgrid without power flow between $\mathrm{AC}$ and $\mathrm{DC}$ buses in the hybrid AC/DC microgrid to simplify the analysis and control schemes of DC sub-microgrid. Usually, DC microgrid should equip with energy storage components, otherwise, the variable DC sources would lead to unstable power supply. However, even equipped with energy storage, the DC sources still need to share their output power to stabilize the DC grid voltage. Therefore, the proper power sharing control schemes implemented in DC microgrid have so far been deeply investigated, which will be reviewed in this section to facilitate the derivation of control schemes for hybrid AC/DC microgrid under islanding operational condition.

Power sharing control scheme for DC microgrid can be generally classified as centralized control scheme and distributed control scheme. The centralized control scheme 


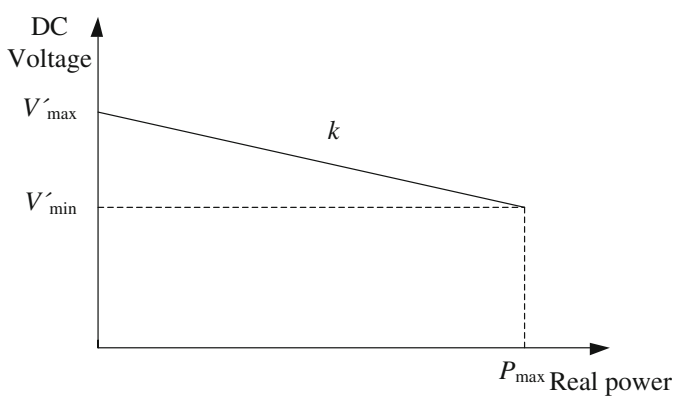

Fig. 3 Real power droop characteristic for DC sub-microgrid

captures the system information by a central controller and generates the corresponding operational commands. Nevertheless, the method depends on the communication between distributed sources and central controller, which definitely will induce additional investment on the communication infrastructure and reduce the reliability of control system [23, 24]. As a consequence, the distributed methods are proposed to eliminate the disadvantage of centralized control scheme. A typical example of distributed control scheme is DC bus signaling (DBS) method [25-27], in which the bus voltage is regarded as global indicator to determine the operational modes of converters according to the defined voltage thresholds. Droop control has been proved to be an effective method to merge multiple sources and storages for proportionally sharing load according to their ratings using only local information. Because DC microgrid does not have reactive power and frequency, the droop control method used in DC sub-microgrid only needs to control the real power sharing by carefully considering converter output voltage and line resistance. In order to achieve proper power sharing, the droop control scheme regulates the output voltage magnitude of distributed converters by using the following equation [28-30].

$V^{\prime}=V_{\max }^{\prime}-k P$

where $V_{\max }^{\prime}$ is the maximum voltage of interfacing converter without load condition; $k$ is the coefficient of droop controller; $P$ is the output real power. The droop coefficient $k$ is introduced for representing the drooping gradient, as drawn in Fig. 3.

$k=\frac{V_{\max }^{\prime}-V_{\min }^{\prime}}{P_{\max }}$

where $V_{\min }^{\prime}$ is the allowable minimum voltage of DC source and $P_{\max }$ is the maximum real power of DC source, respectively.

When this control scheme implemented in several distributed DC sources, (5) can lead to proportional real power sharing if their droop coefficients are regulated according to (6): $k_{1} P_{\max 1}=k_{2} P_{\max 2}=\cdots=k_{N} P_{\max N}$

where $k_{1}$ to $k_{N}$ are the droop coefficients of DC sources 1 to $N ; P_{\max 1}$ to $P_{\max N}$ are the rated real power of DG units 1 to $N$, respectively.

In order to make the DC sub-microgrid more flexible and expandable, the hierarchical control strategy presented in [22] can be assumed either. In specific, hierarchical control principle can be divided into three levels either. The primary level control is responsible for DC bus voltage stability. When the local loads are connected to the DC bus, DC droop controller will obtain equal or proportional DC load current sharing. The secondary control is aiming to eliminate the DC bus voltage deviation introduced by the droop controller. After guaranteeing the performance of DC bus operation by means of primary and secondary level controllers, the tertiary level control is employed to realize the connection to external DC systems. Being similarly, the hierarchical control scheme for DC microgrid still needs communication to achieve the superior operational features of accurate power sharing and enhanced reliability.

\section{Control schemes of hybrid AC/DC microgrid}

Different control methods of hybrid AC/DC microgrids have been presented in the literatures. Droop control scheme was employed in [11], where the controllable loads with different capacities were taken into account. A coordinate control method for a hybrid microgrid composed of various kinds of renewable energy sources was proposed in [31], where detailed models of PV modules, batteries and wind turbines were derived and the energy management strategy for whole system was developed. A configuration with both DC- and AC-links and the corresponding control method were presented in [32], where the DC-link could integrate the local converters with $\mathrm{DC}$ couplings and connect to the common AC-link through DC-AC interfacing converters. A power quality enhancement method was proposed in [33], where the unbalanced and nonlinear loads were taken into account and the control strategies were developed for a multi-bus microgrid. The above-summarized methods can effectively enhance the performance of a hybrid microgrid. But they mainly focus on the AC side performance and the corresponding control schemes.

For the islanding operation of hybrid AC/DC microgrid, the control schemes mentioned in Sections 3 and 4 allow the distributed sources to share the load demands in their respective sub-microgrid. But sharing the load demands in both AC and DC sub-microgrids simultaneously cannot be simply realized by means of droop controlled distributed sources. The power sharing in both sub-microgrids would heavily depend on the control strategy of interlinking 


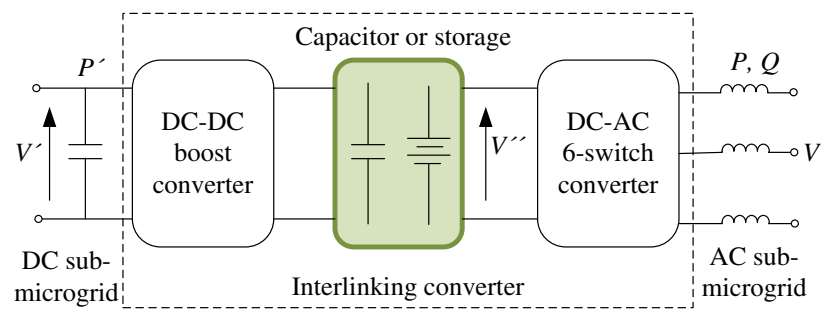

Fig. 4 Topology of interlinking converter

converter. References [30] and [34-36] proposed an autonomous control scheme for the interlinking converter whose responsibility was to link $\mathrm{AC}$ and $\mathrm{DC}$ sub-microgrids together to form the hybrid AC/DC microgrid, where the distributed AC and DC sources were classified into two consolidated sources tied to the same interlinking converter, as shown in Fig. 1. Generally, the interlinking converter consists of a standard DC-DC boost converter and a standard DC-AC inverter, as shown in Fig. 4. $V, V^{\prime}$ and $V^{\prime \prime}$ are the voltages measured at the AC and DC terminals of interlinking converter, and the DC-link capacitor or storage, respectively. $P, Q$ and $P^{\prime}$ are the power flow through the interlinking converter between $\mathrm{AC}$ and $\mathrm{DC}$ microgrids, respectively. Such topology allows the AC and DC voltages in both sub-microgrids to be flexibly controlled, meanwhile allows bidirectional real power flow between both sub-microgrids. At the common DC-link, either capacitor or energy storage can be added for buffering, filtering or storage. According to the configuration of interlinking converter, the control scheme of interlinking converter can be classified as general control scheme, control scheme with DC-link capacitor and control scheme with energy storage.

\subsection{General control scheme}

In AC sub-microgrid, the real power sharing is influenced by the controlled frequency as already addressed in Section 3 , however, the real power sharing in DC sub-microgrid is affected by the controlled voltage as mentioned in Section 4. It is obvious that the droop variables assumed in two sub-microgrids are totally different. Therefore, both droop variables should be properly merged before using them to control the real power across the interfacing converter. A normalized expression has been proposed to normalize the frequency in AC sub-microgrid and the voltage in DC submicrogrid so that their respective ranges of variations commonly span from -1 to 1 [30], i.e.:

$$
\left\{\begin{array}{l}
f_{p u}=\frac{f-0.5\left(f_{\max }+f_{\min }\right)}{0.5\left(f_{\max }-f_{\min }\right)} \\
V_{p u}^{\prime}=\frac{V-0.5\left(V_{\max }^{\prime}+V_{\min }^{\prime}\right)}{0.5\left(V_{\max }^{\prime}-V_{\min }^{\prime}\right)}
\end{array}\right.
$$

where subscripts max and min represent the maximum and minimum values of AC side frequency $f$ and DC side voltage $V^{\prime}$, and subscript pu represents their normalized per-unit values.

As shown in Fig. 5, the AC terminal voltages of interlinking converter are measured to give a phase locked loop (PLL), whose outputs are the AC side frequency $f$, voltage amplitude $V$ and phase angle $\theta$ for $d-q$ transformation. Then, the AC side frequency from PLL and the measured voltage of DC side are normalized by (7). The error of normalized variables $\left(f_{p u}-V_{p u}^{\prime}\right)$ can be fed to a proportional-integral $\left(\mathrm{PI}_{1}\right)$ controller to produce the control reference. In the steady state, the two normalized variables will be forced to be equal, thus the error would be zero [34]. Based on such equalization, the two sub-microgrids would share real power according to their respective overall ratings as illustrated in Fig. 6. This proposal is similar to have a common frequency in the aforementioned AC sub-microgrid, upon which the AC sources can share power proportional to their individual power ratings. The output of $\mathrm{PI}_{1}$ controller is the real power reference $P^{*}$ that should be transferred from DC to AC sub-microgrid through the interfacing converter when it is positive and vice versa. In order to produce the current reference for both sides of interlinking converter, the determined real power reference $P^{*}$ from $\mathrm{PI}_{1}$ controller is converted to a real current reference $I_{d}^{*}$ for AC side and another current reference $I^{* *}$ for DC side, as depicted in Fig. 5 by (8).

$\left\{\begin{array}{l}I_{d}^{*}=\frac{2 P^{*}}{3 V} \\ I^{*}=\frac{-P^{*}}{V^{\prime}}\end{array}\right.$

where $V$ and $V^{\prime}$ are the voltage amplitudes measured at the AC and DC terminals of interlinking converter, respectively.

At the same time, this control scheme can realize reactive power control. The reactive power $Q^{*}$ and the reactive current reference $I_{q}^{*}$ are calculated by measuring the AC terminal voltage $V$ of interlinking converter generated from PLL and using below expressions.

$\left\{\begin{array}{l}Q^{*}=\frac{\left(V-V_{\max }\right)}{n} \\ I_{q}^{*}=\frac{-2 Q^{*}}{3 V}\end{array}\right.$

where $n$ is the droop coefficient for reactive power sharing.

It is noted that only AC sub-microgrid needs the reactive power, which means the reactive current reference $I_{q}^{*}$ calculated by (9) is not applicable when the power transfer from AC sub-microgrid to DC sub-microgrid through the interlinking converter. In the above control scheme, therefore, the 


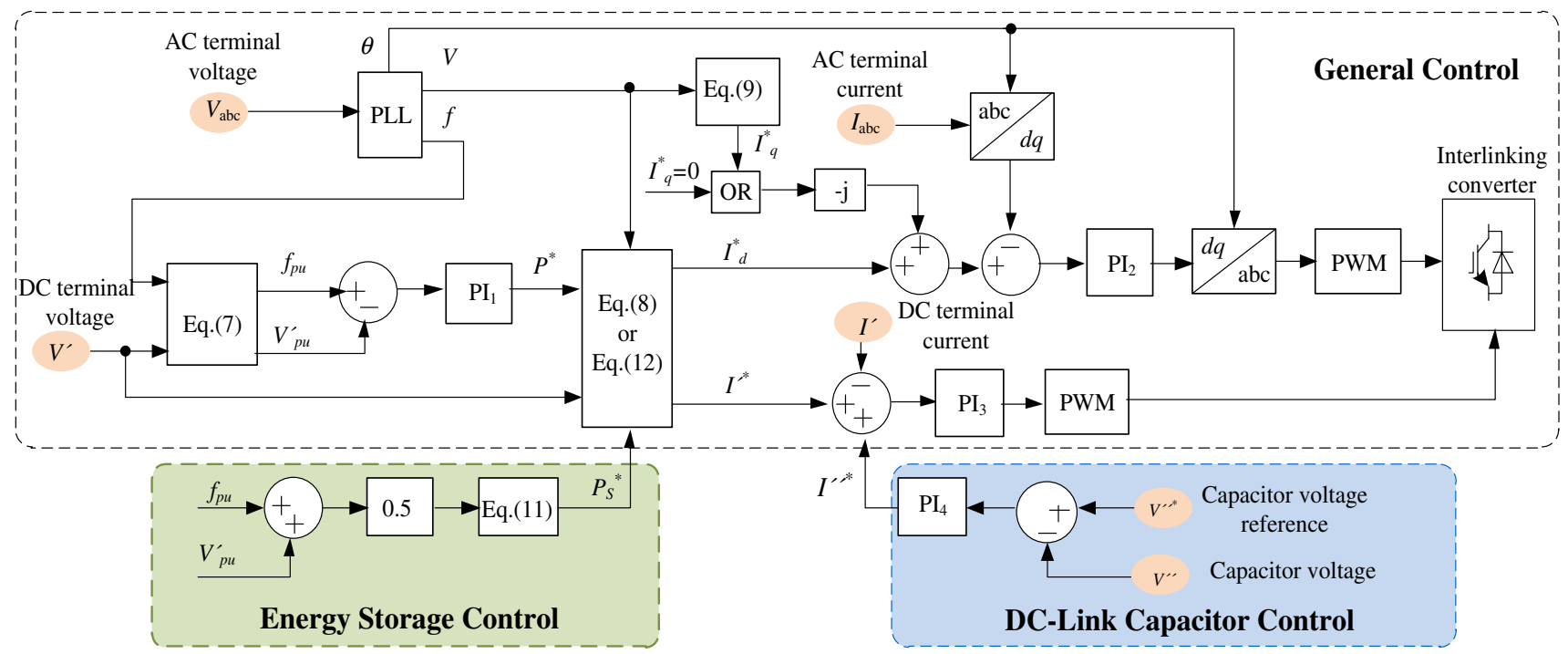

Fig. 5 Control scheme for interlinking converter
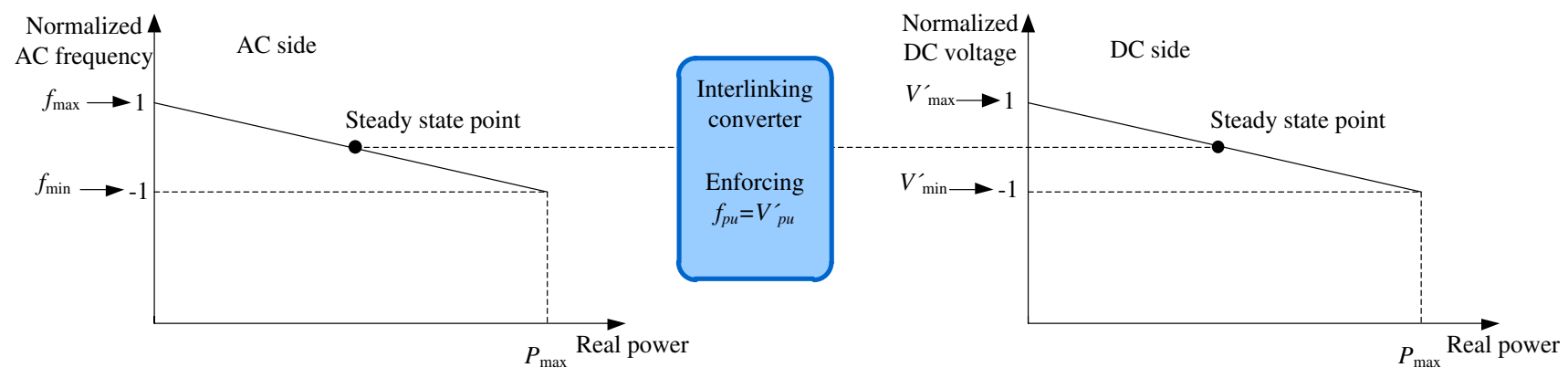

Fig. 6 Illustration of proportional power-sharing process realized within a hybrid microgrid

nonzero $I_{q}^{*}$ calculated by (9) should only be used when real power reference $P^{*}$ is positive. When the real power reference $P^{*}$ is negative, which means the power transfers from AC sub-microgrid to DC sub-microgrid, the reactive current reference $I_{q}^{*}$ should be set to zero for unity power factor operation [35], as shown in Fig. 5. The current references generated for the AC and DC terminals of interlinking converter can be formed as $\left(I_{d}^{*}+\mathrm{j} I_{q}^{*}\right)$ and $I^{*}$.

For the AC side, together with the phase angle $\theta$ from PLL, the measured AC terminal currents are transformed from the stationary frame into the synchronous frame by means of the Park transformation. The combined $d-q$ current references, together with the measured $d-q$ currents, are delivered to proportional-integral $\left(\mathrm{PI}_{2}\right)$ controller, which can achieve proper tracking performance in the synchronous reference frame. On the other hand, a proportional-integral $\left(\mathrm{PI}_{3}\right)$ controller is adopted to force the DC side current to track the current reference from (8) exactly. The output of $\mathrm{PI}_{2}$ and $\mathrm{PI}_{3}$ are the desired modulation signals, which are used to generate the pulse width modulation (PWM) sequences for both side converters.
It is noted that the distributed sources within each submicrogrid still can share the real or reactive power proportionally since they are controlled by the established droop control method reviewed in Section 3 and 4, respectively. The interlinking converter only functions as an energy buffer to control the power flow between $\mathrm{AC}$ and DC sub-microgrids.

\subsection{Control scheme with DC-link capacitor}

In the case of a DC-link capacitor added to the interlinking converter the same as the general AC-DC conversion circuits, the above general control scheme should be further specified. A proportional-integral $\left(\mathrm{PI}_{4}\right)$ controller is introduced to the general control scheme to keep the DClink voltage constant by generating a real current reference $I^{\prime \prime *}$ for compensating the losses in the power conversion circuit, as depicted in Fig. 5. This current reference can be added to the DC current reference of DC side drawn by the DC-DC boost converter from the DC sub-microgrid. 
Meanwhile, the current reference for AC terminal of the interlinking converter remains unchanged.

\subsection{Control scheme with energy storage}

For case, where the energy storage instead of a capacitor is added to the DC-link of interlinking converter, the control scheme of interlinking converter should be revised to fully utilize the energy storage capacity. It means that the energy storages should be charged only when the sources can generate additional energy after meeting the load demands. On the other hand, it should discharge only when the sources cannot fully satisfy the load demands.

The two operation modes require sensing the additional generation capacities of sources. This task can be realized by calculating the DC terminal voltage $V_{p u}^{\prime}$ and AC side frequency $f_{p u}$ as shown in (7) [36], whose values reflect the power flow in $\mathrm{AC}$ and $\mathrm{DC}$ sub-microgrids. Their mean value of $v_{\text {ave }}$ can then be calculated by (10) before substituting it into (11) to generate the charging or discharging power reference $P_{S}^{*}$. Figure 7 illustrates the designed behavior of charging or discharging power of energy storage according to (11).

$v_{\text {ave }}=\frac{f_{p u}+V_{p u}^{\prime}}{2}$

$$
\left\{\begin{array}{l}
P_{S}^{*}=\left\{\begin{array}{l}
P_{S \_ \text {min }}, v_{\text {ave }} \geq v_{t} \\
h\left(v_{\text {ave }}-v_{\mathrm{z}}\right),-1 \leq v_{\text {ave }} \leq v_{t} \\
P_{S \_ \text {max }}, v_{\text {ave }}<-1
\end{array}\right. \\
P_{S \_ \text {min }}<0, \text { Charging } \\
P_{S \_ \text {max }}>0, \text { Discharging } \\
h=\frac{P_{S \_ \text {min }}-P_{S \_ \text {max }}}{1+v_{t}} \\
v_{z}=\frac{P_{S \_\min }+P_{S \_\max } v_{t}}{P_{S \_ \text {max }}-P_{S \_\min }}
\end{array}\right.
$$

where $h$ is the droop coefficient; $v_{t}$ is a boundary value and $v_{z}$ is a middle value at which the energy storage will not absorb or generate real power; $P_{S}^{*}, P_{S_{-} \min }$ and $P_{S_{-} \max }$ are

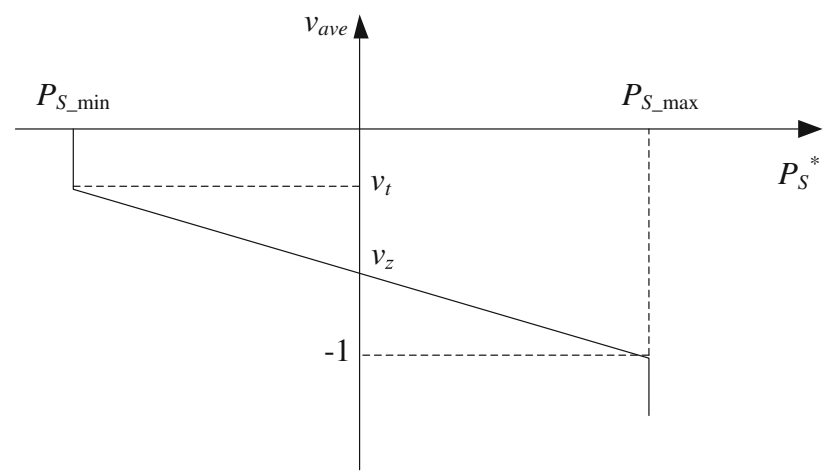

Fig. 7 Energy storage charging and discharging control the real power reference, maximum charging and discharging power of energy storage, respectively. These parameters should be properly set according to the state of charge of energy storage.

The charged/discharged real power of energy storage should be shared in proportion to their rated power or equally in both sub-microgrids. With the energy storage, the AC and DC side real current references calculated by (8) should be changed to those calculated from (12), as shown in Fig. 5. At the same time, the reactive current reference remains unchanged.

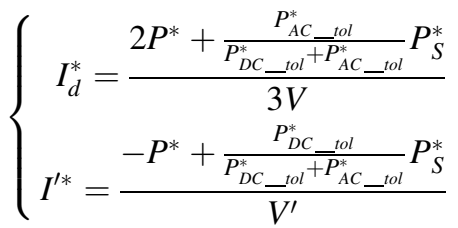

or

$$
\left\{\begin{array}{c}
I_{d}^{*}=\frac{2 P^{*}+0.5 P_{S}^{*}}{3 V} \\
I^{\prime *}=\frac{-P^{*}+0.5 P_{S}^{*}}{V^{\prime}}
\end{array}\right.
$$

where $I_{d}^{*}$ and $I^{*}$ are the real current reference for AC side converter and the current reference for DC side converter of interlinking converter; $P^{*}$ is the real power reference transferred between AC and DC sub-microgrids; $P_{S}^{*}$ is the energy storage real power reference; $P_{D C_{-} t o l}^{*} P_{A C_{-} t o l}^{*}$ are the total real power ratings of $\mathrm{DC}$ and $\mathrm{AC}$ microgrids, respectively.

\section{Conclusion}

With recent interests in reliable and economical power delivery, hybrid AC/DC microgrids have been conceived as practical and effective solutions. Robust control of a hybrid microgrid encounters many challenges. This paper reviews hybrid AC/DC microgrid control methods under islanding operational condition. The operational features and detailed control schemes of AC sub-microgrid, DC sub-microgrid and hybrid AC/DC microgrid are reviewed. The control schemes can effectively manage the power flow between AC and DC sub-microgrids and achieve the proper power sharing and enhance operational reliability.

Open Access This article is distributed under the terms of the Creative Commons Attribution License which permits any use, distribution, and reproduction in any medium, provided the original author(s) and the source are credited.

\section{References}

[1] Blaabjerg F, Zhe C, Kjaer SB (2004) Power electronics as efficient interface in dispersed power generation systems. IEEE Trans Power Electron 19(5):1184-1194 
[2] Carrasco JM, Franquelo LG, Bialasiewwicz JT et al (2006) Powerelectronic systems for the grid integration of renewable energy sources: a survey. IEEE Trans Power Electron 53(4):1002-1016

[3] Lasseter RH, Paigi P (2004) Microgrid: a conceptual solution. In: Proceedings of the 2004 IEEE power electronics specialists conference, vol. 6, Aachen, Germany 20-25 Jun 2004, pp 4285-4290

[4] Sao CK, Lehn PW (2008) Control and power management of converter fed MicroGrids. IEEE Trans Power Syst 23(3): 1088-1098

[5] Baran ME, Mahajan NR (2003) DC distribution for industrial systems: opportunities and challenges. IEEE Trans Ind Appl 39(6):1596-1601

[6] Sannino A, Postiglione G, Bollen MHJ (2003) Feasibility of a DC network for commercial facilities. IEEE Trans Ind Appl 39(5):1409-1507

[7] Mahmoodi M, Noroozian R, Gharehpetian GB et al (2007) A suitable power transfer control system for interconnection converter of DC microgrids. In: Proceedings of the 2007 international conference on renewable energies and power quality, vol. 6, Sevilla 28-30 March 2007, pp 2231-2237

[8] Ito Y, Yang Z, Akagi H (2004) DC microgrid based distribution power generation system. In: Proceedings of the 4th international power electronics and motion control conference (IPEMC), vol. 3, Xi' an China, 14-16 Aug 2004, pp 1740-1745

[9] Xiao JF, Wang P (2013) Multiple modes control of household DC microgrid with integration of various renewable energy sources. In: The 39th annual conference of the IEEE industrial electronics society (IECON-2013), Vienna, Austria, 10-13 Nov 2013, pp 1773-1778

]10] Wang P, Goel L, Liu X, Choo FH (2013) Harmonizing AC and DC: a hybrid AC/DC future grid solution. IEEE Power Energy Mag 11(3):76-83

[11] Kurohane K, Senjyu T, Yona A et al (2010) A hybrid smart AC/ DC power system. IEEE Trans Smart Grid 1(2):199-204

[12] Brabandere KD, Bolsens B, Keybus JVD et al (2007) A voltage and frequency droop control method for parallel inverters. IEEE Trans Power Electron 22(4):1107-1115

[13] Prodanovic M, Green TC (2006) High-quality power generation through distributed control of a power park microgrid. IEEE Trans Ind Electron 53(5):1471-1482

[14] Marwali MN, Jung JW, Keyhani A (2004) Control of distributed generation systems: part II. Load sharing control. IEEE Trans Power Electron 19(6):1551-1561

[15] Martins AP, Carvalho AS, Araujo AS (1995) Design and implementation of a current controller for the parallel operation of standard upss. In: Proceedings of the 1995 IEEE IECON, 21st international conference on industrial electronics, control, and instrumentation, vol. 1 Orlando, FL, USA, 6-10 Nov 1995, pp 584-589

[16] Chen JF, Chu CL (1995) Combination voltage-controlled and current-controlled PWM inverters for UPS parallel operation. IEEE Trans Power Electron 10(5):547-558

[17] Wu TF, Chen YK, Huang YH (2000) 3C strategy for inverters in parallel operation achieving an equal current distribution. IEEE Trans Ind. Electron 47(2):273-281

[18] Sun X, Lee YS, Xu DH (2003) Modeling, analysis, and implementation of parallel multi-inverter systems with instantaneous average-current-sharing scheme. IEEE Trans Power Electron 18(3):844-856

[19] Guerrero JM, Matas J, de Vicuña LG et al (2006) Wirelesscontrol strategy for parallel operation of distributed generation inverters. IEEE Trans Ind Electron 53(5):1461-1470

[20] Zhang Q (2013) Robust droop controller for accurate proportional load sharing among inverters operated in parallel. IEEE Trans Ind Electron 60(4):1281-1290
[21] He JW, Li YW (2011) Analysis, design and implementation of virtual impedance for power electronics interfaced distributed generation. IEEE Trans Ind Appl 47(6):2525-2538

[22] Guerrero JM, Vasquez JC, Matas J et al (2011) Hierarchical control of droop-controlled ac and dc microgrids- a general approach toward standardization. IEEE Trans Ind Electron 58(1): $158-172$

[23] Xianghui C, Jiming C, Yang X, Youxian S (2010) Buildingenvironment control with wireless sensor and actuator networks: Centralized versus distributed. IEEE Trans Ind Electron 57(11): 3596-3605

[24] Wang BC, Sechilariu M, Locment F (2012) Intelligent DC microgrid with smart grid communications: control strategy consideration and design. IEEE Trans Smart Grid 3(4):2148-2156

[25] Sun K, Zhang L, Xing Y, Guerrero JM (2011) A distributed control strategy based on DC bus signaling for modular photovoltaic Generation systems with battery Energy storage. IEEE Trans Power Electron 26(10):3032-3045

[26] Schonberger J, Duke R, Round SD (2006) DC-bus signaling: a distributed control strategy for a hybrid renewable nanogrid. IEEE Trans Ind Electron 53(5):1453-1460

[27] Zhang L, Wu TJ, Xing Y et al (2011) Power control of DC microgrid using DC bus signaling. In: Proceedings of twenty-sixth annual IEEE applied power electronics conference and exposition, Fort Worth, TX, USA, 6-11 Mar 2011, pp 1926-1932

[28] Johnson BK, Lasseter RH, Alvarado FL, Adapa R (1993) Expandable multiterminal dc systems based on voltage droop. IEEE Trans Power Deliv 8(4):1926-1932

[29] Noroozian R et al (2010) Grid-tied and stand-alone operation of distributed generation modules aggregated by cascaded boost converters. J Power Electron 10(1):97-105

[30] Jin J, Loh PC, Wang P, Mi Y, Blaabjerg F (2010) Autonomous operation of hybrid ac-dc microgrids. In: Proceedings 2010 IEEE international conference on sustainable energy technologies (ICSET), Kandy Sri Lanka, 6-9 Dec 2010, pp 1-7

[31] Liu X, Wang P, Loh PC (2011) A hybrid AC/DC microgrid and its coordination control. IEEE Trans Smart Grid 2(2):278-286

[32] Jiang Z, Yu X (2008) Hybrid dc- and ac-linked microgrids: towards integration of distributed energy resources. In: Proceedings of the IEEE energy 2030 conference, Atlanta, GA, USA, 17-18 Nov. 2008, 8pp

[33] Shahnia F, Majumder R, Ghosh A et al (2010) Operation and control of a hybrid microgrid containing unbalanced and nonlinear loads. Electric Power Syst Res 80(8):954-965

[34] Loh PC, Li D, Chai YK, Blaabjerg F (2013) Autonomous operation of hybrid microgrid with ac and dc subgrids. IEEE Trans Power Electron 28(5):2214-2223

[35] Loh PC, Blaabjerg F (2011) Autonomous control of distributed storages in microgrids. In: Proceedings of the IEEE 8th international conference on power electronics and ECCE Asia, Jeju, South Korea, 30 May-3 Jun 2011, pp 536-542

[36] Loh PC, Li D, Chai YK et al (2013) Autonomous control of interlinking converter with energy storage in hybrid ac-dc microgrid. IEEE Trans Ind Appl 49(3):1374-1382

Guangqian DING (S'13) received the B.Eng. degree in electronic information engineering from Shandong University of Science and Technology, Qingdao, China, in 2004, and the M.Eng. degree in electrical engineering from Shandong University, Jinan, China, in 2010. From 2004 to 2005, he was an Assistant Engineer with LG Innotek Huizhou Co., Ltd, China. From 2005 to 2012, he was a project manager with Shandong electric power school, China. He is currently pursuing his $\mathrm{Ph} . \mathrm{D}$. degree in electrical engineering from Shandong University, Jinan, China. His research interests include 
power electronics on distributed power systems, microgrid, and power quality.

Feng GAO (S'07-M'09) received the B.Eng. and M.Eng. degrees in electrical engineering from Shandong University, Jinan, China, in 2002 and 2005, respectively, and the Ph.D. degree from the School of Electrical and Electronic Engineering, Nanyang Technological University, Singapore, in 2009. From 2008 to 2009, he was a research fellow in Nanyang Technological University. Since 2010, he joined the School of Electrical Engineering, Shandong University, where he is currently a professor and serving as Vice Dean. From September 2006 to February 2007, he was a visiting scholar at the Institute of Energy Technology, Aalborg University, Aalborg, Denmark. Dr. GAO was the recipient of the IEEE Industry Applications Society Industrial Power Converter Committee Prize for a paper published in 2006, and he is now serving as an Associate Editor of the IEEE Transactions on Power Electronics.

Song ZHANG received the B.Eng. degree in electrical engineering from Shandong University of Science and Technology, Qingdao, China, in 2004, and the M.Eng. degree in electrical engineering from Shandong University, Jinan, China, in 2011. From 2004 to 2011, she was a teacher with Shandong electric power school, Taian, China. Since 2012, she was a teacher with State Grid of China Technology College, Jinan, China. Her research interests include the power system stability and control, power system protection and microgrid.

Poh Chiang LOH (S'01-M'04-SM'12) received the B.Eng. degree (Hons.) and the M.Eng. degree from the National University of Singapore, Singapore, in 1998 and 2000, respectively, and the Ph.D. degree from Monash University, Melbourne, Vic., Australia, in 2002, all in electrical engineering. During the summer of 2001, he was a Visiting Scholar with the Wisconsin Electric Machine and Power Electronics Consortium, University of Wisconsin-Madison, where he was involved in the synchronized implementation of cascaded multilevel inverters, and reduced common-mode carrier-based and hysteresis control strategies for multilevel inverters. From 2002 to
2003, he was a Project Engineer with the Defence Science and Technology Agency, Singapore, managing defence infrastructure projects and exploring new technology for defence applications. From 2003 to 2009, he was an Assistant Professor with the Nanyang Technological University, Singapore, and since 2009, he has been an Associate Professor at the same university. In 2005, he has been a Visiting Staff first at the University of Hong Kong, and then at Aalborg University, Aalborg, Denmark. In 2007 and 2009, he again returned to Aalborg University first as a Visiting Staff working on matrix converters and the control of grid-interfaced inverters, and then as a Guest Member of the Vestas Power Program. Dr. LOH has received two third paper prizes from the IEEE-IAS IPCC committee in 2003 and 2006, and he is now serving as an Associate Editor of the IEEE Transactions on Power Electronics.

Frede BLAABJERG (F'03) received the Ph.D. degree from Aalborg University, Aalborg, Denmark, during 1988-1992. He was with ABBScandia, Randers, Denmark, from 1987 to 1988. He became an Assistant Professor in 1992, an Associate Professor in 1996, and a Full Professor in power electronics and drives in 1998 at Aalborg University. He has been a Part-Time Research Leader at Research Center Risoe in wind turbines. During 2006-2010, he was the Dean of the Faculty of Engineering, Science, and Medicine and became a Visiting Professor at Zhejiang University, Zhejiang, China, in 2009. His research interests include power electronics and its applications such as wind turbines, PV systems, and adjustable speed drives. Dr. BLAABJERG has been the Editor in Chief of the IEEE Transactions on Power Electronics since 2006. He was a Distingueshed Lecturer for the IEEE Power Electronics Society during 2005-2007 and for the IEEE Industry Applications Society during 2010-2011. He received the 1995 Angelos Award for his contribution in modulation technique and the Annual Teacher prize at Aalborg University. In 1998, he received the Outstanding Young Power Electronics Engineer Award from the IEEE Power Electronics Society. He has received ten IEEE Prize paper awards and another prize paper award at PELINCEC Poland 2005. He received the IEEE PELS Distingueshed Service Award in 2009 and the EPE-PEMC 2010 Council award. 